\title{
Light Microscopic Analysis for Bone Responses to Implant Surfaces.
}

\author{
In-Sung Yeo ${ }^{1}$, Jae-Hyuk Sim $^{2}$ and Taek-Ka Kwon ${ }^{3}$ \\ 1. Department of Prosthodontics, School of Dentistry and Dental Research Institute, Seoul National \\ University, Seoul, Korea. \\ 2. Department of Prosthodontics, Seoul National University School of Dentistry, Seoul, Korea. \\ 3. Department of Dentistry, St. Catholic Hospital, Catholic University of Korea, Suwon, Korea.
}

Clinically, dental implants are an excellent treatment option for missing teeth. Implant surfaces have been modified in various ways in order to accelerate biologic bone responses around the implants, resulting in the decrease of patients' edentulous periods. Histomorphometry by light microscope is known to be the most reliable method to evaluate the early biologic response at the interface between the bone and the implant surface. The purpose of the study was to investigate the early bone response to several modified implant surfaces using histomorphometric analysis by light microscope. The tibiae of New Zealand white rabbits received the modified surfaced screw-shaped titanium implants; two implants in each tibia. The first experiment compared among the calcium phosphate coated, anodized, and sandblasted surfaces; the second between the calcium phosphate and hydroxyapatite coated surfaces; the third between fluoride-treated and anodized surfaces; the last among calcium phosphate coated/anodized (CPA), sandblasted/acid-etched (SA), and anodized surfaces. Commercially pure titanium surfaced implants served as control. The rabbits were sacrificed after 2 weeks of the implant insertion. The tibial bone blocks were sectioned and processed in the undemineralized states for the microscopic analyses. Two histomorphometric quantities were measured; bone-to-implant contact (BIC), which is defined as ratio of the bone length contacted with the implant surface over the total length of the surface, and bone area (BA), which is defined as ratio of the bone formed between the implant threads over the total area between the threads. The modified surfaces investigated in the study showed higher BIC and BA than the control $(\mathrm{P}<0.05)$. The CPA surface suggested higher BA than the anodized surface $(\mathrm{P}<0.05)$. There were no significant differences in the rest of comparisons among the modified surfaces. In conclusion, modified implant surfaces demonstrate the accelerated bone responses from the results of microscopic histomorphometry, compared to the commercially pure titanium surface. These microscopic analyses may be adequate tools to evaluate early bone responses to implant surfaces. However, such analyses have the limit of 2-dimensional measurement. This field requires an improved micro-analysis technique for 3-dimensional evaluation. 

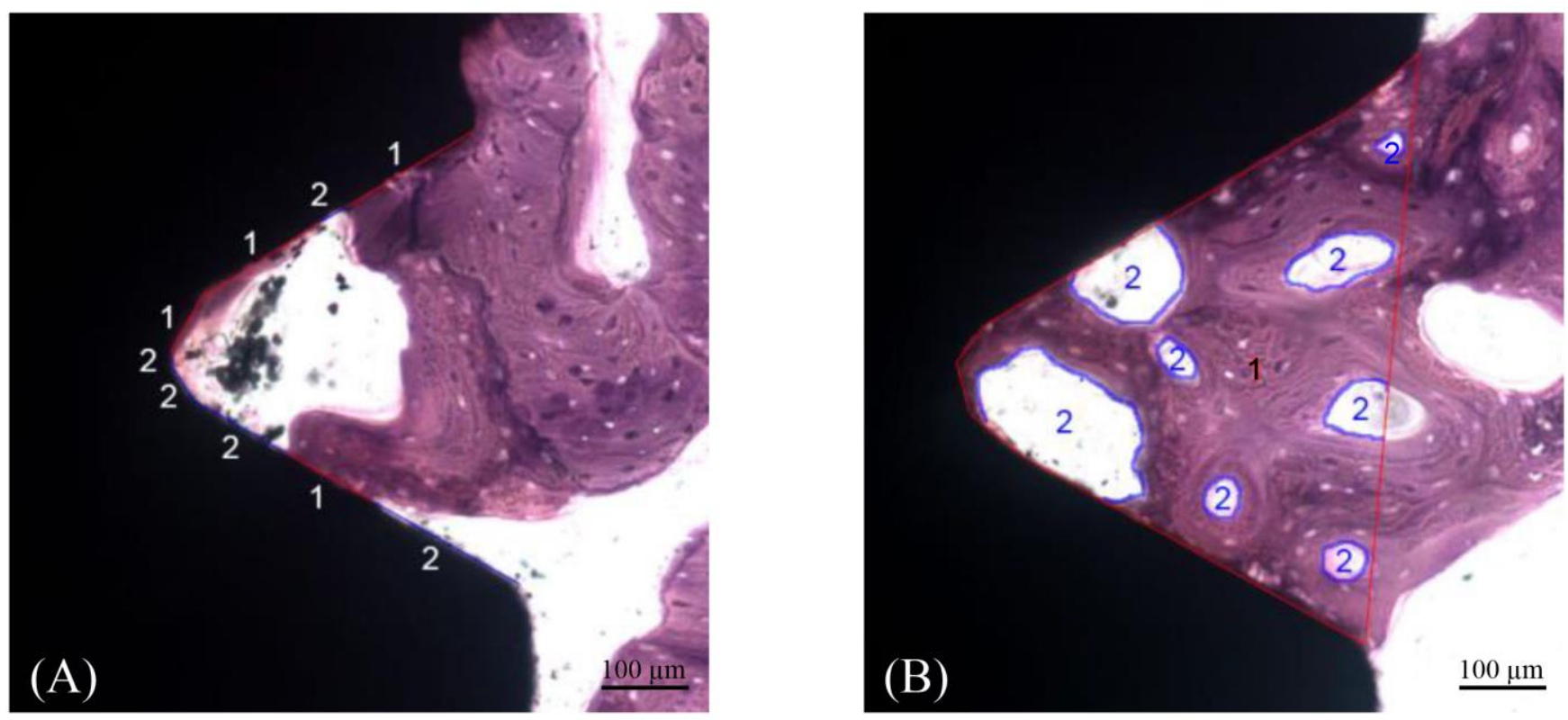

Figure 1. The measurements of the histomorphometric quantities. (A) The parts of the implant that the bone attaches with are designated as 1 (red lines), and those of the implant without bone contact are designated as 2 (blue lines). BIC is calculated as (the sum of 1s) / (the sum of $1 \mathrm{~s}$ and 2s). (B) The total area between the implant threads is designated as 1 (in the red line polygon), and the areas without bone are designated as 2 . BA is calculated as $\{$ (the area of 1$)-($ the sum of $2 \mathrm{~s})\} /$ (the area of 1 ).

\section{References:}

[1] Yeo IS, et al, Journal of Biomedical Materials Research Part B: Applied Biomaterials, 87 (2008), p. 303.

[2] Yeo IS, et al, Journal of the Korean Physical Society, 57 (2010), p. 1717.

[3] Choi JY, et al, Implant Dentistry, 21 (2012), p. 124.

[4] Koh JW, et al, The International Journal of Oral \& Maxillofacial Implants, 28 (2013), p. 790. 